\title{
Research Regarding Dynamics of Chemical Content from Pasteurized Egg Melange Stored in Polyethylene Type Packings
}

\author{
ROXANA NICOLETA RATU, MARIUS GIORGI USTUROI, DANIEL SIMEANU, CRISTINA SIMEANU, ALEXANDRU USTUROI, \\ MARIUS GHEORGHE DOLIS* \\ University of Agricultural Sciences and Veterinary Medicine of lasi, 3 M. Sadoveanu Alley, 700490, Iasi, Romania
}

\begin{abstract}
In the current paper, we aimed to analyze the way in which packing (different polyethylene types) influence the quality of pasteurized melange during storage, packing being realized in units of $1 \mathrm{~kg}$ (Tetra Pak) (batch Lexp-1) and units of $5 \mathrm{~kg}$ (Bag in box) (batch Lexp-2). Products were stored during a period of 28 days at a temperature of $+4^{\circ} \mathrm{C}$, qualitative determinations being realized in first day (day 0), at 7 days, at 14 days, 21 days and in day 28 of storage. Were effectuated a sensorial examination and chemical analysis were was established the content in dry matter (\%), water (\%), proteins (\%), content in essential amino acids (isoleucine, methionine, tryptophan, phenylalanine) and non-essential amino acids (alanine, histidine, glycine, serine) $(\mathrm{mg} / \mathrm{100g})$ as well as the content in lipids (\%) establishing their profile by identification of some saturated fatty acids (16:0 mg/100g and 18:0 mg/100g) and unsaturated fatty acids $(16: 1 \mathrm{mg} / 100 \mathrm{~g}$ and $18: 1 \mathrm{mg} /$ 100g). After sensorial examinations, the first modifications were observed at the checking effectuated in day 21 for batch Lexp-2, the obtained score being of 18 points, and at checking effectuated in day 28 was given a score of 18 points for melange belonging to batch Lexp-1 and only 14 points for melange from batch Lexp-2. Differences were recorded also in case of chemical composition of products, so for protein content at batch Lexp-1 in first checking day was obtained a mean of $12.730 \pm 0.24 \%$ and at batch Lexp-2 $12.614 \pm 0.22 \%$. Differences between those two batches were insignificant $(p<0.05)$. In case of fat content, at the end of storage period was obtained a mean of $11.256 \pm 0.06 \%$ for batch Lexp-1 and $11.244 \pm 0.11 \%$ for batch Lexp-2, differences being insignificant $(p<0.05)$. Regarding the profile of amino acids and fatty acids, the mean values obtained during whole storage period oscillated from one stage to another, but the differences between those two batches were insignificant $(p<0.05)$. Pasteurized egg melange suffers certain sensorial modifications during storage, especially on consistency and colouring, modifications which are accentuated mainly bystorage conditions. Type of polyethylene utilized for this product hadn't influenced the nutritive qualities of product
\end{abstract}

Keywords: chemical composition, pasteurized eggs, polyethylene, storage

Advances in food processing and food packaging playa primary role in keeping the U.S. food supply among the safest in the world. Simply stated, packaging maintains the benefits of food processing after the process is complete, enabling foods to travel safely for long distances from their point of origin and still be wholesome at the time of consumption. However, packaging technology must balance food protection with other issues, including energy and material costs, heightened social and environmental consciousness, and strict regulations on pollutants and disposal of municipal solid waste [1].

The principal roles of food packaging are to protect food products from outside influences and damage, to contain the food, and to provide consumers with ingredient and nutritional information [2].

Eggs have been classified as nature's original functional food [3-7]. Eggs are one of the most complete foods for human consumption because they are rich in vitamins, minerals, fatty acids, and proteins that provide several essential amino acids of excellent biological value [8-12].

In case of eggs sold in shell, even if were tested several preservation methods, the best results were obtained by refrigeration, method utilized, nowadays, in majority of world's avian units [13, 14].

Even if proved its efficiency in time, storage of eggs in refrigeration conditions could generate severe problems, mostly when aren't respected storage conditions (especially thermal level), delivery conditions (refrigerated eggs must be gradually warmed) or quality conditions of eggs subjected to refrigeration (dirty eggs, with broken shell, stale etc.) [15-18].

In the food industry, egg products (dried pasteurized egg, pasteurized egg) are used preferentially rather than fresh eggs (shell eggs), because in addition to retaining flavor, color, nutritional value, and functional properties, they offer advantages such as better uniformity, less storage space, and ease of portion measurement $[19,20]$.

Also by pasteurization process is destroyed a great part of microbial charge, Salmonella enterica sevor Enteritis being the pathogen bacteria which could generate severe infections among consumers, egg being the most common product which could caused this contamination [21-23].

The term Egg Products refers to processed or convenience forms of eggs obtained by breaking and processing shell eggs. Egg products include whole eggs, egg whites, and egg yolks in frozen, refrigerated liquid, and dried forms available in a number of different product formulations, as well as specialty egg products.

In recent years, food industry prefers broken and pasteurized eggs for use as liquid whole egg, liquid white and liquid yolk $[24,25]$.

Package design and construction plays a significant role in determining the shelf life of a food product. The right selection of packaging materials and technologies maintains product quality and freshness during distribution and storage $[26,27]$.

Egg pasteurized products are sold in plastic bottles (PET), in Tetra Pak boxes or in packing units type Bag in box, all of those being on the market in different quantities. 
Plastics are made by condensation polymerization (polycondensation) or addition polymerization (poly-addition) of monomer units. In poly-condensation, the polymer chain grows by condensation reactions between molecules and is accompanied by formation of low molecular weight byproducts such as water and methanol [28, 29].

There are two major categories of plastics: thermosets and thermoplastics [30]. Thermosets are polymers that solidify or set irreversibly when heated and cannot be remolded. Because they are strong and durable, they tend to be used primarily in automobiles and construction applications such as adhesives and coatings, not in food packaging applications. On the other hand, thermoplastics are polymers that soften upon exposure to heat and return to their original condition at room temperature. Because thermoplastics can easily be shaped and molded into various products such as bottles, jugs, and plastic films, they are ideal for food packaging [31, 32].

Polyolefin is a collective term for polyethylene and polypropylene, the two most widely used plastics in food packaging, and other less popular olefin polymers. Both polyethylene and polypropylene possess a successful combination of properties, including flexibility, strength, lightness, stability, moisture and chemical resistance, and easy processing, and are well suited for recycling and reuse. The simplest and most inexpensive plastic made by addition polymerization of ethylene is polyethylene. There are two basic categories of polyethylene: high density and low density. High-density polyethylene is stiff, strong, tough, resistant to chemicals and moisture, permeable to gas, easy to process, and easy to form. Low-density polyethylene is flexible, strong, tough, easy to seal, and resistant to moisture. Because low-density polyethylene is relatively transparent, it is predominately used in film applications and in applications where heat sealing is necessary [33].

So, in the current paper we aimed to analyze the influence of polyethylene type, utilized in packing, on the pasteurized and packed egg melange during storage.

\section{Experimental part}

\section{Material and method}

Biological material was represented by pasteurized egg melange (without additives and preservatives) packed in units of $1 \mathrm{~kg}$ (Tetra Pak) (batch Lexp-1) and units of $5 \mathrm{~kg}$ (Bag in box) (batch Lexp-2). Products were achieved directly from the producer in the processing day ( 5 pieces Tetra Pak and 5 pieces Bag in box) and stored during 28 days at a temperature of $+4^{\circ} \mathrm{C}$. Qualitative determinations were realized in the first day (day 0), at 7 days, at 14 days, 21 days and in day 28 of storage. We mention the fact that at each qualitative check stages were unwrapped new packs.

Were tracked the sensorial characteristics of product $(500 \mathrm{~mL} / \mathrm{batch} / \mathrm{check})$, and were analyzed the evolution of aspect, consistency, smell and taste. Sensorial characteristics were determined based on scale points system, characterized by the fact that for each feature are established 6 quality levels, mark 0 being given to a product with severe modifications or even altered. At each check stage were gathered samples from those two products and were given to team formed by five members. Appreciation of samples' sensorial features was realized by comparing with the existent standard quality conditions [34].

Quality chemical indicators $(500 \mathrm{~mL} / \mathrm{lot} / \mathrm{check})$, were determined on dried product, melange being dehydrated at $60^{\circ} \mathrm{C}$ in a forced airflow incubator, Memmert IFE 500 model, for 48 hours. On dried product were made analysis to determine content in dry matter $(\%)$, water $(\%)$, proteins (\%) (total nitrogen) establishing also the contentin essential amino acids (isoleucine, methionine, tryptophan, phenylalanine) and non-essential amino acids (alanine, histidine, glycine, serine) $(\mathrm{mg} / \mathrm{l} 00 \mathrm{~g})$ as well as content of lipids (\%) establishing also their profile by identification of some saturated fatty acids (16:0 mg/100g and $18: 0 \mathrm{mg} /$ $100 \mathrm{~g})$ and non-saturated fatty acids (16:1 mg/100g and $18: 1 \mathrm{mg} / 100 \mathrm{~g})$.

Water content (W\%) was established as difference in according with the formula: Water $(\%)=100 \%-\mathrm{DM}(\%)$ $[35,36]$.

Content in dry matter (DM\%) was established through AOAC no. 952.30 method [37] which is based on pre-dried eggs by dehydration in a MemmertUFE 700 forced air oven at $100^{\circ} \mathrm{C}$.

Crude protein (CP\%) resulted from total nitrogen content assessment via the Kjeldahl method, applied on a Velp Scientifica DK 6 digestion and UDK 7 distillation system, according to AOAC no. 925.31 method [38].

Quantitative determination of amino acids was effectuated using the method described in literature [39] and using high performance amino acid analyzer for the separation of amino acids, while tryptophan was colourimetrically determinate according to the method described also in literature [40].

Total lipids as crude fat (CF) content was determined by AOAC method no 925.32 [41], using a Velp Scientifica Soxhlet SER 148 extractor.

Fatty acids was established through AOAC no. 971.11 [42] method with GC Carlo Erba 5300 mega series Gaschromatograph and a sample chromatogram with resulting fatty acid.

Collected data were subjected to statistical computation, using the ANOVA one-way algorithm included in MsExcel, to calculate the descriptive statistics (mean, standard error) and find out whether there were significant differences and upgraded with PostHoc Daniel's XL Toolbox version 4.01 (http://xltoolbox.sf.net), to identify the differences [43].

\section{Results and discussions}

In table 1 are presented the results of sensorial examination realized on pasteurized melange and wrapped in different polyethylene types. Analysis effectuated on fresh products (day 0 ) and after that at 7 days and 14 storage days didn't show modifications of the tracked parameters, each batch being appreciated with the maximum score (20 points), being as a homogenous paste, with agglomerations and foreigner bodies; smell and taste being characteristically pleasant.

The first modification were observed in day 21 of checking and in day 28 was obtained a score of 18 points for product belonging to batch Lexp-1 and only 14 points for product from batch Lexp-2.

To determine crude chemical composition was necessary to establish content in water (\%) in dry matter (\%) (table 1).

Regarding water content (\%) the mean values calculated by us enlightened a slow decreasing from one check stage to another for each studied batch, differences between batches being insignificant during whole period. So, at batch Lexp-1 in day 0 of checking was obtained a mean value of $75.312 \pm 0.02 \%$ with a minimum of $75.25 \%$ while the maximum value was $75.39 \%$. The studied character presented a very good homogeneity, value of variation coefficient being of only $0.069 \%$. For batch Lexp- 
Table 1

SENSORIAL CHARACTERISTICS OF PASTEURIZED MELANGE

\begin{tabular}{|c|c|c|c|c|c|c|c|}
\hline \multirow[b]{2}{*}{$\begin{array}{l}\text { Period } \\
\text { (days) }\end{array}$} & \multirow[b]{2}{*}{$\begin{array}{c}\text { Analyzed } \\
\text { assortment }\end{array}$} & \multirow[b]{2}{*}{$\begin{array}{c}\text { Experimental } \\
\text { batch }\end{array}$} & \multicolumn{5}{|c|}{ Sensorial characteristics and obtained score } \\
\hline & & & Aspect & Consistency & Smell and taste & Color & $\begin{array}{c}\text { Total number } \\
\text { of points }\end{array}$ \\
\hline \multirow{2}{*}{$\mathbf{0}$} & \multirow{2}{*}{ Melange } & Lexp-1 & 5 & 5 & 5 & 5 & 20 \\
\hline & & Lexp-2 & 5 & 5 & 5 & 5 & 20 \\
\hline \multirow{2}{*}{7} & \multirow{2}{*}{ Melange } & Lexp-1 & 5 & 5 & 5 & 5 & 20 \\
\hline & & Lexp-2 & 5 & 5 & 5 & 5 & 20 \\
\hline \multirow{2}{*}{14} & \multirow{2}{*}{ Melange } & Lexp-1 & 5 & 5 & 5 & 5 & 20 \\
\hline & & Lexp-2 & 5 & 5 & 5 & 5 & 20 \\
\hline \multirow{2}{*}{21} & \multirow{2}{*}{ Melange } & Lexp-1 & 5 & 5 & 5 & 5 & 20 \\
\hline & & Lexp-2 & 4 & 5 & 4 & 5 & 18 \\
\hline \multirow{2}{*}{28} & \multirow{2}{*}{ Melange } & Lexp-1 & 4 & 4 & 5 & 5 & 18 \\
\hline & & Lexp-2 & 3 & 3 & 4 & 4 & 14 \\
\hline
\end{tabular}

\begin{tabular}{|c|c|c|c|c|c|}
\hline \multirow{2}{*}{$\begin{array}{c}\text { Period } \\
\text { (days) }\end{array}$} & \multirow{2}{*}{ Batch } & \multicolumn{4}{|c|}{ Statistic estimators } \\
\cline { 3 - 6 } & & $X \pm s_{\bar{X}}$ & V\% & Minimum & Maximum \\
\hline \multirow{2}{*}{$\mathbf{0}$} & Lexp-1 & $75.312 \pm 0.02^{\mathrm{a}}$ & 0.069 & 75.25 & 75.39 \\
\cline { 2 - 6 } & Lexp-2 & $75.330 \pm 0.03^{\mathrm{a}}$ & 0.100 & 75.23 & 75.41 \\
\hline \multirow{2}{*}{7} & Lexp-1 & $75.294 \pm 0.05^{\mathrm{a}}$ & 0.149 & 75.11 & 75.39 \\
\cline { 2 - 6 } & Lexp-2 & $75.298 \pm 0.03^{\mathrm{a}}$ & 0.100 & 75.19 & 75.37 \\
\hline \multirow{2}{*}{$\mathbf{1 4}$} & Lexp-1 & $75.230 \pm 0.04^{\mathrm{a}}$ & 0.119 & 75.09 & 75.31 \\
\cline { 2 - 6 } & Lexp-2 & $75.260 \pm 0.03^{\mathrm{a}}$ & 0.103 & 75.15 & 75.34 \\
\hline \multirow{2}{*}{$\mathbf{2 1}$} & Lexp-1 & $75.180 \pm 0.02^{\mathrm{a}}$ & 0.084 & 75.11 & 75.26 \\
\cline { 2 - 6 } & Lexp-2 & $75.204 \pm 0.03^{\mathrm{a}}$ & 0.098 & 75.09 & 75.28 \\
\hline \multirow{2}{*}{$\mathbf{2 8}$} & Lexp-1 & $75.134 \pm 0.04^{\mathrm{a}}$ & 0.127 & 75 & 75.25 \\
\cline { 2 - 6 } & Lexp-2 & $75.130 \pm 0.03^{\mathrm{a}}$ & 0.123 & 74.98 & 75.20 \\
\hline
\end{tabular}

ANOVA within rows, between groups for different superscripts, one by one comparison: aa: not significant; ab significant, $*(P<0.05)$; distinguished significant $=\mathrm{ac}, * *(P<0.01)$; highly significant $=$ ad *** $(P<0.001)$

2 minimum was placed at a level of $75.23 \%$ and in this case maximum value was $75.41 \%$, mean being of $75.330 \pm 0.03 \%$. Also in this case was enlightened a very good homogeneity, variation coefficient value being $0.100 \%$.

Situation was similar at the following effectuated checking's, so at the end of experiment (day 28 of storage), water content from analyzed product recorded mean values of $75.134 \pm 0.04 \%$ for batch Lexp-1 and $75.130 \pm 0.03 \%$ for batch Lexp-2. At batch level it is a good homogeneity of studied character, aspect confirmed by the very low values of variation coefficient of $0.127 \%$ respectively $0.123 \%$.

Similar with water content from product the dry matter content was quite constant during experiment, reason to explain the lack of differences with statistical significance between batches, at each effectuated check.
So, for samples belonging to batch Lexp-1, rate of DM varied between $24.688 \pm 0.02$ recorded before storage (fresh product) and $24.866 \pm 0.04 \%$ at the end of storage period (day 28). For this batch, the studied character presented a very good homogeneity, values of variation coefficient being of only $0.211-0.454 \%$ (table 3 ).

Chemical composition of pasteurized melange isn't influenced only by storage period, so the main components could suffer modifications only if pasteurization wasn't done properly or if products weren't stored at right temperature [44 - 47].

Regarding protein contentfor batch Lexp-1 in firstcheck day (day 0 ) values oscillated between $11.80 \%$ and $13.11 \%$ calculated mean being $12.730 \pm 0.24 \%$. For variation coefficient, we obtained a value of $4.198 \%$, fact which indicates a very homogenous character inside batch. For batch Lexp-2 mean was $12.614 \pm 0.22 \%$ and variation

\begin{tabular}{|c|c|c|c|c|c|}
\hline \multirow{2}{*}{$\begin{array}{c}\text { Period } \\
\text { (days) }\end{array}$} & \multirow{2}{*}{ Batch } & \multicolumn{4}{|c|}{ Statistic estimators } \\
\cline { 3 - 6 } & & $X \pm s_{\bar{X}}$ & V\% & Minimum & Maximum \\
\hline \multirow{2}{*}{$\mathbf{0}$} & Lexp-1 & $24.688 \pm 0.02^{\mathrm{a}}$ & 0.211 & 24.61 & 24.75 \\
\cline { 2 - 6 } & Lexp-2 & $24.670 \pm 0.03^{\mathrm{a}}$ & 0.305 & 24.59 & 24.77 \\
\hline \multirow{2}{*}{7} & Lexp-1 & $24.706 \pm 0.05^{\mathrm{a}}$ & 0.454 & 24.61 & 24.89 \\
\cline { 2 - 6 } & Lexp-2 & $24.702 \pm 0.03^{\mathrm{a}}$ & 0.306 & 24.63 & 24.81 \\
\hline \multirow{2}{*}{$\mathbf{1 4}$} & Lexp-1 & $24.770 \pm 0.04^{\mathrm{a}}$ & 0.362 & 24.69 & 24.91 \\
\cline { 2 - 6 } & Lexp-2 & $24.740 \pm 0.03^{\mathrm{a}}$ & 0.314 & 24.66 & 24.85 \\
\hline \multirow{2}{*}{$\mathbf{2 1}$} & Lexp-1 & $24.821 \pm 0.03^{\mathrm{a}}$ & 0.256 & 24.74 & 24.89 \\
\cline { 2 - 6 } & Lexp-2 & $24.796 \pm 0.03^{\mathrm{a}}$ & 0.297 & 24.72 & 24.91 \\
\hline \multirow{2}{*}{28} & Lexp-1 & $24.866 \pm 0.04^{\mathrm{a}}$ & 0.384 & 24.75 & 25.00 \\
\cline { 2 - 6 } & Lexp-2 & $24.870 \pm 0.04^{\mathrm{a}}$ & 0.372 & 24.80 & 25.02 \\
\hline
\end{tabular}

Table 3

EVOLUTION OF DRY MATTER CONTENT (\%) FROM PASTEURIZED MELANGE AND PACKED IN DIFFERENT

POLYETHYLENE TYPES

ANOVA within rows, between groups for different superscripts, one by one comparison: aa: not significant; ab significant, $*(P<0.05)$; distinguished significant $=\mathrm{ac}, * *(P<0.01)$;

highly significant $=$ ad $* * *(P<0.001)$. 
coefficient value was $3.931 \%$, which also indicates a very good homogeneity inside batch. After analysis, at this first check, weren't enlightened differences with statistical significance (table 4).

In day 28 of storage the obtained means were of $12.740 \pm 0.16 \%$ for batch Lexp-1 and of $12.688 \pm 0.09 \%$ for batch Lexp-2, differences being also insignificant (table 4).

Regarding the profile of amino acids the highest values for essential amino acids were founded for phenylalanine where for Lexp-1 in first check day (day 0) we obtain a mean value of $650.4 \pm 0.51 \mathrm{mg} / 100 \mathrm{~g}$ and in day 28 of checking the mean value was $650.6 \pm 0.93 \mathrm{mg} / 100 \mathrm{~g}$. In case of batch Lexp-2 the obtained mean values were lower, but the observed differences didn't present statistical significance; 50 , in first storage day (day 0) mean obtained by us was of $650.0 \pm 0.63 \mathrm{mg} / 100 \mathrm{~g}$, in day 7 of $649.8 \pm 0.80$ $\mathrm{mg} / 100 \mathrm{~g}$, for the analysis effectuated in day 14 of storage was obtained a mean of $650.2 \pm 0.66 \mathrm{mg} / 100 \mathrm{~g}$, for day 21 of storage the mean was $650.4 \pm 0.51 \mathrm{mg} / 100 \mathrm{~g}$ and the value of $650.0 \pm 0.95 \mathrm{mg} / 100 \mathrm{~g}$ was recorded in the last storage day, respectively day 28 (table 5 ).
Non-essential amino acids analyzed on egg melange were represented by alanine, histidine, glycine and serine. The obtained values after calculus of statistical estimators enlightened the highest values for content in serine, where means for batch Lexp-1 in first storage day were $900.35 \pm 0.25 \mathrm{mg} / 100 \mathrm{~g}$ and $893.88 \pm 2.43 \mathrm{mg} / 100 \mathrm{~g}$ for batch Lexp-2 (differences between those two batches being insignificant), values which in day 28 of checking reached the means of $900.44 \pm 0.2 \mathrm{mg} / 100 \mathrm{~g}$ for batch Lexp1 and $891.30 \pm 1.0 \mathrm{mg} / 100 \mathrm{~g}$ for batch Lexp-2.

Lipids are founded only in yolk being represented by triglycerides $66 \%$ from total lipids, phospholipids $28 \%$ from total lipids and cholesterol 5\% [48] .

Regarding fat content for batch Lexp-1 in first check day (day 0) values oscillated between $10.89 \%$ and $11.47 \%$ calculated mean being $11.294 \pm 0.10 \%$. In case of batch Lexp-2 maximum reached till $11.244 \pm 0.11 \%$, value obtained in day 28 and $11.206 \pm 0.12 \%$ value obtained in day 7 (table 6).

In the case of batches studied by us, regarding the content in saturated fatty acids, such as content in palmitic acid (16:0) and stearic acid (18:0), were recorded similar values from one check stage to another, the observed

Table 4

EVOLUTION OF PROTEIN CONTENT (\%) FROM PASTEURIZED MELANGE AND PACKED IN DIFFERENT POLYETHYLENE TYPES

\begin{tabular}{|c|c|c|c|c|c|c|c|c|}
\hline \multirow{2}{*}{$\begin{array}{c}\text { Period } \\
\text { (days) }\end{array}$} & \multicolumn{4}{|c|}{ Batch Lexp-1 } & \multicolumn{4}{c|}{ Batch Lexp-2 } \\
\cline { 2 - 10 } & $\bar{X} \pm s_{\bar{X}}$ & V\% & Min. & Max. & $\bar{X} \pm s_{\bar{X}}$ & V\% & Min. & Max. \\
\hline $\mathbf{0}$ & $12.730 \pm 0.24^{\mathrm{a}}$ & 4.198 & 11.80 & 13.11 & $12.614 \pm 0.22^{\mathrm{a}}$ & 3.931 & 11.88 & 13.25 \\
\hline $\mathbf{7}$ & $12.772 \pm 0.22^{\mathrm{a}}$ & 3.767 & 11.95 & 13.16 & $12.676 \pm 0.14^{\mathrm{a}}$ & 2.549 & 12.41 & 13.19 \\
\hline $\mathbf{1 4}$ & $12.726 \pm 0.18^{\mathrm{a}}$ & 3.207 & 12.02 & 13.02 & $12.626 \pm 0.13^{\mathrm{a}}$ & 2.370 & 12.22 & 12.99 \\
\hline $\mathbf{2 1}$ & $12.710 \pm 0.18^{\mathrm{a}}$ & 3.084 & 12.05 & 13.01 & $12.694 \pm 0.09^{\mathrm{a}}$ & 1.508 & 12.5 & 13.01 \\
\hline $\mathbf{2 8}$ & $12.740 \pm 0.16^{\mathrm{a}}$ & 2.767 & 12.30 & 13.10 & $12.688 \pm 0.09^{\mathrm{a}}$ & 1.505 & 12.50 & 12.99 \\
\hline
\end{tabular}

ANOVA within rows, between groups for different superscripts, one by one comparison:

aa: not significant; ab significant, $*(P<0.05)$; distinguished significant $=a c$,

$* *(P<0.01)$; highly significant $=$ ad $* * *(P<0.001)$.

\begin{tabular}{|c|c|c|c|c|c|c|}
\hline \multirow{2}{*}{ Batch } & \multirow{2}{*}{ Indicator } & \multicolumn{5}{|c|}{ Period (days) } \\
\hline & & 0 & 7 & 14 & 21 & 28 \\
\hline \multicolumn{7}{|c|}{ Essential amino acids, from which: } \\
\hline Lexp-1 & \multirow{2}{*}{ Isoleucine } & $592.6 \pm 2.25^{\mathrm{a}}$ & $591.8 \pm 3.84^{2}$ & $592 \pm 3.75^{\mathrm{a}}$ & $592.4 \pm 2.86^{\mathrm{a}}$ & $592.6 \pm 1.63^{\mathrm{a}}$ \\
\hline Lexp-2 & & $591.8 \pm 1.93^{\mathrm{a}}$ & $591.4 \pm 3.82^{2}$ & $591.6 \pm 3.61^{\mathrm{a}}$ & $591.6 \pm 3.34^{\mathrm{a}}$ & $591.8 \pm 1.46^{\mathrm{a}}$ \\
\hline Lexp-1 & \multirow{2}{*}{ Methionine } & $426.6 \pm 1.89^{\mathrm{a}}$ & $425.8 \pm 2.22^{2}$ & $425.6 \pm 1.29^{\mathrm{a}}$ & $426.2 \pm 1.07^{\mathrm{a}}$ & $426.2 \pm 0.97^{\mathrm{a}}$ \\
\hline Lexp-2 & & $426.0 \pm 2.10^{\mathrm{a}}$ & $425.6 \pm 2.11^{2}$ & $424.8 \pm 1.59^{\mathrm{a}}$ & $424.6 \pm 1.75^{\mathrm{a}}$ & $425.0 \pm 1.14^{\mathrm{a}}$ \\
\hline Lexp-1 & \multirow{2}{*}{ Tryptophan } & $201.4 \pm 0.51^{\mathrm{a}}$ & $201.2 \pm 0.86^{2}$ & $201.0 \pm 1.05^{\mathrm{a}}$ & $201.2 \pm 0.58^{\mathrm{a}}$ & $201.6 \pm 0.68^{\mathrm{a}}$ \\
\hline Lexp-2 & & $201.0 \pm 0.32^{\mathrm{a}}$ & $200.8 \pm 0.80^{2}$ & $200.6 \pm 0.68^{a}$ & $201.0 \pm 0.45^{\mathrm{a}}$ & $201.4 \pm 0.51^{\mathrm{a}}$ \\
\hline Lexp-1 & \multirow{2}{*}{ Phenylalanine } & $650.4 \pm 0.51^{\mathrm{a}}$ & $650.2 \pm 0.66^{2}$ & $650.4 \pm 0.51^{\mathrm{a}}$ & $650.8 \pm 0.37^{\mathrm{a}}$ & $650.6 \pm 0.93^{\mathrm{a}}$ \\
\hline Lexp-2 & & $650.0 \pm 0.63^{\mathrm{a}}$ & $649.8 \pm 0.80^{2}$ & $650.2 \pm 0.66^{\mathrm{a}}$ & $650.4 \pm 0.51^{\mathrm{a}}$ & $650.0 \pm 0.95^{\mathrm{a}}$ \\
\hline \multicolumn{7}{|c|}{ Non-essential amino acids, from which: } \\
\hline Lexp-1 & \multirow{2}{*}{ Alanine } & $720.19 \pm 0.25 a$ & $720.08 \pm 0,24^{\mathrm{a}}$ & $720.13 \pm 0,31^{\mathrm{a}}$ & $719.82 \pm 0,22^{\mathrm{a}}$ & $720.25 \pm 0,1^{\mathrm{a}}$ \\
\hline Lexp-2 & & $720.16 \pm 0.06 a$ & $720.01 \pm 0,20^{\mathrm{a}}$ & $720.10 \pm 0,31^{\mathrm{a}}$ & $719.92 \pm 0,16^{a}$ & $720.15 \pm 0,0^{\mathrm{a}}$ \\
\hline Lexp-1 & \multirow{2}{*}{ Histidine } & $342.05 \pm 0.03 a$ & $342.04 \pm 0.04^{\mathrm{a}}$ & $342.14 \pm 0.37^{\mathrm{a}}$ & $341.97 \pm 0.31^{\mathrm{a}}$ & $342.44 \pm 0.2^{\mathrm{a}}$ \\
\hline Lexp-2 & & $341.05 \pm 0.43 \mathrm{a}$ & $341.10 \pm 0.38^{\mathrm{a}}$ & $340.99 \pm 0.56^{\mathrm{a}}$ & $341.42 \pm 0.18^{\mathrm{a}}$ & $341.19 \pm 0.0^{\mathrm{a}}$ \\
\hline Lexp-1 & \multirow{2}{*}{ Glycine } & $410.54 \pm 0.18 \mathrm{a}$ & $410.83 \pm 0.13^{\mathrm{a}}$ & $411.05 \pm 0.08^{\mathrm{a}}$ & $410.42 \pm 0.18^{\mathrm{a}}$ & $411.05 \pm 0.1^{\mathrm{a}}$ \\
\hline Lexp-2 & & $409.03 \pm 0.33 a$ & $408.81=0.24^{\mathrm{a}}$ & $409.01 \pm 0.07^{\mathrm{a}}$ & $408.80 \pm 0.29^{a}$ & $409.00=0.0^{\mathrm{a}}$ \\
\hline Lexp-1 & \multirow{2}{*}{ Serine } & $900.35 \pm 0.25 a$ & $900.22 \pm 0.25^{\mathrm{a}}$ & $900.14 \pm 0.33^{\mathrm{a}}$ & $899.92 \pm 0.67^{a}$ & $900.44 \pm 0.2^{\mathrm{a}}$ \\
\hline Lexp-2 & & $893.88 \pm 2.43 \mathrm{a}$ & $892.56 \pm 3.89^{\mathrm{a}}$ & $893.74 \pm 1.02^{\mathrm{a}}$ & $892.46 \pm 0.28^{\mathrm{a}}$ & $891.30 \pm 1.0^{\mathrm{a}}$ \\
\hline
\end{tabular}

Table 5

EVOLUTION OF AMINO ACIDS CONTENT (mg/ 100g) FROM PASTEURIZED MELANGE AND PACKED IN DIFFERENT POLYETHYLENE TYPES

ANOVA within rows, between groups for different superscripts, one by one comparison: aa: not significant; ab significant,

$*(P<0.05)$; distinguished significant $=$ ac, $* *(P<0.01)$; highly significant $=$ ad $* * *(P<0.001)$. 
Table 6

EVOLUTION OF FAT CONTENT (\%) FROM PASTEURIZED MELANGE AND PACKED IN DIFFERENT POLYETHYLENE TYPES

\begin{tabular}{|c|c|c|c|c|c|c|c|c|}
\hline \multirow{2}{*}{$\begin{array}{c}\text { Period } \\
\text { (days) }\end{array}$} & \multicolumn{4}{|c|}{ Batch Lexp-1 } & \multicolumn{4}{c|}{ Batch Lexp-2 } \\
\cline { 2 - 10 } & $X \pm s_{\bar{X}}$ & $\mathrm{~V} \%$ & Min. & Max. & $X \pm s_{\bar{X}}$ & V\% & Min. & Max. \\
\hline 0 & $11.294 \pm 0.10^{\mathrm{a}}$ & 2.071 & 10.89 & 11.47 & $11.228 \pm 0.12$ & 2.406 & 10.89 & 11.44 \\
\hline 7 & $1.244 \pm 0.09^{\mathrm{a}}$ & 1.825 & 10.90 & 11.40 & $11.206 \pm 0.12$ & 2.391 & 10.87 & 11.42 \\
\hline 14 & $11.238 \pm 0.08^{\mathrm{a}}$ & 1.619 & 10.98 & 11.41 & $11.214 \pm 0.12$ & 2.360 & 10.90 & 11.42 \\
\hline $\mathbf{2 1}$ & $11.308 \pm 0.08^{\mathrm{a}}$ & 1.609 & 11.11 & 11.59 & $11.248 \pm 0.12$ & 2.358 & 10.94 & 11.53 \\
\hline $\mathbf{2 8}$ & $11.256 \pm 0.06^{\mathrm{a}}$ & 1.095 & 11.05 & 11.35 & $11.244 \pm 0.11$ & 2.196 & 10.95 & 11.50 \\
\hline
\end{tabular}

ANOVA within rows, between groups for different superscripts, one by one comparison: aa: not significant; ab significant, $*(P<0.05)$; distinguished significant $=\mathrm{ac}, * *(P<0.01)$; highly significant $=$ ad $* * *(P<0.001)$.

Table 7

EVOLUTION OF LIPID COMPONENTS (g/100g) FROM PASTEURIZED MELANGE AND PACKED IN DIFFERENT POLYETHYLENE TYPES

\begin{tabular}{|c|c|c|c|c|c|c|}
\hline \multirow{2}{*}{ Batch } & \multirow{2}{*}{ Indicator } & \multicolumn{5}{|c|}{ Period (days) } \\
\hline & & 0 & 7 & 14 & 21 & 28 \\
\hline \multicolumn{7}{|c|}{ Saturated fatty acids, from which: } \\
\hline Lexp-1 & \multirow{2}{*}{ 16:0 palmitic acid } & $2.172 \pm 0.01^{2}$ & $2.183 \pm 0.02^{2}$ & $2.190 \pm 0.02^{2}$ & $2.189 \pm 0.01^{\mathrm{a}}$ & $2.184 \pm 0.01^{3}$ \\
\hline Lexp-2 & & $2.168 \pm 0.01^{2}$ & $2.194 \pm 0.02^{2}$ & $2.177 \pm 0.01^{2}$ & $2.184 \pm 0.01^{\mathrm{a}}$ & $2.180 \pm 0.01^{\mathrm{s}}$ \\
\hline Lexp-1 & \multirow{2}{*}{ 18:0 stearic acid } & $0.730 \pm 0.02^{2}$ & $0.705 \pm 0.01^{2}$ & $0.700 \pm 0.01^{2}$ & $0.707 \pm 0.01^{\mathrm{a}}$ & $0.705 \pm 0.01^{3}$ \\
\hline Lexp-2 & & $0.727 \pm 0.02^{2}$ & $0.704 \pm 0.01^{2}$ & $0.695 \pm 0.01^{2}$ & $0.706 \pm 0.01^{\mathrm{a}}$ & $0.696 \pm 0.01^{a}$ \\
\hline \multicolumn{7}{|c|}{ Unsaturated fatty acids, from which: } \\
\hline Lexp-1 & \multirow{2}{*}{$\begin{array}{c}\text { 16:1 palmitoleic } \\
\text { acid }\end{array}$} & $0.283 \pm 0.01^{2}$ & $0.281 \pm 0.01^{2}$ & $0.274 \pm 0.01^{2}$ & $0.288 \pm 0.01^{\mathrm{a}}$ & $0.278 \pm 0.01^{3}$ \\
\hline Lexp-2 & & $0.289 \pm 0.01^{2}$ & $0.292 \pm 0.01^{2}$ & $0.288 \pm 0.01^{2}$ & $0.290 \pm 0.01^{\mathrm{a}}$ & $0.297 \pm 0.01^{3}$ \\
\hline Lexp-1 & \multirow{2}{*}{ 18:1 oleic acid } & $3.102 \pm 0.16^{2}$ & $3.093 \pm 0.20^{2}$ & $3.209 \pm 0.12^{2}$ & $3.208 \pm 0.07^{\mathrm{a}}$ & $3.248 \pm 0.09^{a}$ \\
\hline Lexp-2 & & $3.274 \pm 0.01^{2}$ & $3.283 \pm 0.05^{2}$ & $3.289 \pm 0.05^{2}$ & $3.191 \pm 0.05^{\mathrm{a}}$ & $3.215 \pm 0.03^{\mathrm{a}}$ \\
\hline
\end{tabular}

ANOVA within rows, between groups for different superscripts, one by one comparison: aa: not significant; ab significant,

$*(P<0.05)$; distinguished significant $=$ ac, $* *(P<0.01)$; highly significant $=$ ad $* * *(P<0.001)$.

differences between those two batches being insignificant. So, for batch Lexp-1 the highest content in 16:0 was recorded at the checking made in day 14 , mean being of $2.190 \pm 0.02 \mathrm{~g} / 100 \mathrm{~g}$ and the lowest level was recorded at checking realized in first day with a value of $2.172 \pm 0.01 \mathrm{~g} /$ $100 \mathrm{~g}$ (table 7).

Regarding content in palmitic acid (16:0) for pasteurized melange and packed in polyethylene bags belonging to batch Lexp-2, calculated mean values were $2.168 \pm 0.01$ $\mathrm{g} / 100 \mathrm{~g}$ for first effectuated check (day 0), $2.194 \pm 0.02 \mathrm{~g} /$ $100 \mathrm{~g}$ at checking effectuated in day 7 of storage, $2.177 \pm 0.01 \mathrm{~g} / 100 \mathrm{~g}$ at 14 days, $2.184 \pm 0.01$ at 21 days of storage and 2.180 \pm 0.01 at last effectuated check (day 28).

For content of stearic acid (18:0) values oscillated between $0.700 \pm 0.01 \mathrm{mg} / 100 \mathrm{~g}$ recorded at check effectuated in day 14 and $0.730 \pm 0.02 \mathrm{mg} / 100 \mathrm{~g}$ value calculated for fresh product (day 0), for pasteurized melange belonging to batch Lexp-1 (table 7).

For palmitoleic acid (16:1) at batch Lexp-1 values oscillated between $0.274 \pm 0.01 \mathrm{mg} / 100 \mathrm{~g}$ value obtained in day 14 and $0.288 \pm 0.01 \mathrm{mg} / 100 \mathrm{~g}$ mean obtained in day 21. In case of batch Lexp-2 maximum reached till $0.297 \pm 0.01 \mathrm{mg} / 100$, value obtained in day 28 and $0.288 \pm 0.01 \mathrm{mg} / 100 \mathrm{~g}$ value obtained in day 14 (table 7).

In the case of unsaturated fatty acids, the highest values were recorded for oleic acid (18:1) as follows $3.102 \pm 0.16$ $\mathrm{mg} / 100 \mathrm{~g}$ for batch Lexp-1 and $3.274 \pm 0.01 \mathrm{mg} / 100 \mathrm{~g}$ for batch Lexp-2 in first day of experiments (day 0). Dynamic analysis of oleic acid revealed the fact that those one kept constant during storage, recorded differences from one check stage to another being insignificant.
In the case of palmitoleic acid (16:1) the obtained values were much lower, so for batch Lexp-1, in first day of determinations (day 0) mean was of $0.283 \pm 0.01 \mathrm{mg} / 100 \mathrm{~g}$ reaching at the end of storage period, day 28 , at a mean of $0.278 \pm 0.01 \mathrm{mg} / 100 \mathrm{~g}$. For batch Lexp-2 we obtained a mean of $0.289 \pm 0.01 \mathrm{mg} / 100 \mathrm{~g}$ in first day, $0.292 \pm 0.01 \mathrm{mg} /$ $100 \mathrm{~g}$ in day $7,0.288 \pm 0.01 \mathrm{mg} / 100 \mathrm{~g}$ in day 14 of storage, $0.290 \pm 0.01 \mathrm{mg} / 100 \mathrm{~g}$ in day 21 and $0.297 \pm 0.01 \mathrm{mg} / 100 \mathrm{~g}$ at last effectuated checking. Must be mentioned the fact that also in this case weren't observed differences with statistical significance.

\section{Conclusions}

After sensorial examination were identified modifications, more accentuated at batch Lexp-2, when after 21 days of storage was recorded a score of 18 points, score for batch Lexp-1 being 20 points. At the last effectuated check, day 28, were enlightened modifications at both products, those ones being more severe for batch Lexp-2 were the score was 14 points and 18 points for batch Lexp-1.

Regarding water content were observed low losses at both batches, but differences didn't present statistical differences $(p<0.05)$. So, at the end of storage period (day 28), the obtained mean value for batch Lexp-1 was $75.134 \pm 0.04 \%$ and for batch Lexp-2 was $75.130 \pm 0.03 \%$.

In case of dry matter content the obtained mean values at the end of storage period were $24.866 \pm 0.04 \%$ for batch Lexp-1 and $24.870 \pm 0.04 \%$ for batch Lexp-2. Studied character presented a very good homogeneity for both batches, differences between them being insignificant $(p<0.05)$. 
Regarding the protein content of melange belonging to batch Lexp-1, the obtained mean value at first check (day 0) was $12.730 \pm 0.24 \%$ reaching at the end of storage period at $12.740 \pm 0.16 \%$. For batch Lexp-2 mean calculated in first storage day was $12.614 \pm 0.22 \%$, mean value calculated in day 28 was $12.688 \pm 0.09 \%$. As in the case of other determinations weren't observed differences with statistical significance $(p<0.05)$ between those two batches during storage period.

Regarding amino acids profile the means obtained by us were $592.6 \pm 2.25 \mathrm{mg} / 100 \mathrm{~g}$ for isoleucine content at batch Lexp-1 in day 0 of checking and $591.8 \pm 1.93 \mathrm{mg} /$ $100 \mathrm{~g}$ at batch Lexp-2 in the same period. In the case of phenylalanine, the recorded mean values at the end of storage period were $650.6 \pm 0.93 \mathrm{mg} / 100 \mathrm{~g}$ for batch Lexp1 and $650.0 \pm 0.95 \mathrm{mg} / 100 \mathrm{~g}$ for batch Lexp-2. From the non-essential amino acids, the highest content was observed for serine, mean of batch Lexp-1 being $900.35 \pm 0.25 \mathrm{mg} / 100 \mathrm{~g}$ at first check effectuated and at batch Lexp-2 mean reached till $893.88 \pm 2.43 \mathrm{mg} / 100 \mathrm{~g}$.

Even if mean values oscillated from one check stage to another both in case of essential amino acids and also for non-essential amino acids, the recorded differences between batches were insignificant $(p<0.05)$.

In case of saturated acids content, the most significant values were obtained for palmitic acid (16:0) where mean at beginning of storage period (day 0) was $2.172 \pm 0.01 \mathrm{~g} /$ $100 \mathrm{~g}$ for batch Lexp-1 and $2.168 \pm 0.01 \mathrm{~g} / 100 \mathrm{~g}$ for batch Lexp-2.

For unsaturated fatty acids, highest values were recorded for oleic acid (18:1) where means at the end of storage period were $3.248 \pm 0.09 \mathrm{~g} / 100 \mathrm{~g}$ for batch Lexp-1 and $3.215 \pm 0.03 \mathrm{~g} / 100 \mathrm{~g}$ for batch Lexp-2. Also in this case weren't observed differences with statistical significance during checking stages $(p<0.05)$.

Lack of differences between batches, regarding chemical composition of product, show the fact that polyethylene type utilized for packing of pasteurized melange didn't influenced the product quality during storage.

\section{References}

1.MARSH, K., BUGUSU, B., Food packaging Roles, materials, and environmental issues, J. Food Sci., vol. 72, 2007, pp. R39-R55.

2.COLES, R., Introduction. In: Coles R, McDowell D, Kirwan MJ, editors. Food packaging technology, London, U.K.: Blackwell Publishing, CRC Press, 2003, pp 1-31.

3.HASLER, C.M., The changing face of functional foods, Journal of the American College of Nutrition, Vol. 19, 2000, pp. 499S-506S.

4.NYS, Y., SAUVEUR, B., Valeur nutritionnelle des œufs, INRA Prod. Anim. vol. 17, no. 5 2004, pp. 385-393

5.BOURRE, J.M., GALEA, F., An important source of omega-3 fatty acids, vitamins $D$ and $E$, carotenoids, iodine and selenium: $A$ new natural multi-enriched egg, J ournal of Nutr. Health Aging, no 10, 2006, pp. 371-376.

6.CHERIAN, G., HOLSONBAKE, T.B., GOEGER, M.P., Fatty acid composition and egg components of specialty eggs, Poultry Science, vol. 81, 2002, pp. 30-33.

7.SURAI, P.F., SPARKS, N.H.C., Designer eggs: from improvement of egg composition to functional food, Trends in Food Science and Technology, 12, 2001, pp. 7-16.

8.SAUVEUR, B., Structure, composition et valeur nutritionnelle de I'oeuf, 347-374; Qualité de I'oeuf, 377-433. In: Reproduction des volailles et production d'oeuf, INRA (eds), 1988, p. 449.

9.NYS, Y., BAIN, M., VAN IMMERSEEL, F., Improving the safety and quality of egg and egg products, Woodhean Publishing Limited, Cambridge, UK, 2011, p. 148.
10.CRESPO, N., ESTEVE-GARCIA, E., Dietary fatty acid profile modifies abdominal fat deposition in broiler chickens, Poultry Science, no. 80, 2001, pp 71-78.

11.BOLUKBASI, S.C., ERHAN, M.K., KAYNAR, O., The effect of feeding thyme, sage and rosemary oil on laying hen performance, cholesterol and some proteins ratio of egg yolk and Escherichia coli count in feces, Arch. Geflugelkd, vol. 72, 2008, pp. 231-237.

12.EBUBEKIR, A., TKERODLU, A., Effect of egg shape index on mechanical properties of chicken eggs, J ournal of Food Engineering, vol. 88, 2008, pp. $606-612$.

13.SHIN, D., NARCISO-GAYTAN, C., REGENSTEIN, J.M., SANCHEZ-PLATA, M.X., Effect of various refrigeration temperatures on quality of shell eggs, J ournal of the Science of Food and Agriculture, Vol. 92, 2012, pp. $1341-1345$.

14.J ONES, D.R., MUSGROVE, M.T., Effect of extended storage on egg quality factors', Poultry Science, vol. 84, 2005, pp. 1774-1777.

15.KAHRAMAN-DOæAN, H., BAYINDIRLI, L., ÖZILGEN, M., Quality control charts for storage of eggs, Food Quality, vol. 17, 1994, pp.495 $-501$.

16.SCOTT, T.A., SILVERSIDES, F.G., The effect of storage and strain of hen on egg quality, Poultry Science, vol. 79, 2000, pp. 1725 - 1729.

17.SILVERSIDES, F.G., SCOTT, T.A., Effect of storage and layer age on quality of eggs from two lines of hens, Poultry Science, vol. 80, 2001, pp.1240 - 1245.

18.ARAGON-ALEGRO, L.C., SOUZA, K.L.O., SOBRINHO, P.S.C., LANDGRAF, M., DESTRO M.T., Avaliação da qualidade microbiologica de ovo integral pasteurizado produzido com e sem a etapa de lavagem no processamento (Influence of washing in the microbial quality of pasteurized egg), Ciênc. Tecnol. Aliment, vol. 25, 2005, pp.618-622. 19.RÊGO, O.P., MENEZES, L.D.M., FIGUEIREDO, T.C., OLIVEIRA, D.D., ROCHA, J.S.R., LARA, L.J.C., LIMA, A.L., SOUZA, M.R., CANCADO, S.V., Bioactive amines and microbiological quality in pasteurized and refrigerated liquid whole egg, Poultry Science, vol. 93, 2014, pp. 1018-1022.

20.HOPE, B.K., BAKER, A.R., EDEL, E.D., HOGUE, A.T., SCHLOSSER, W.D., WHITING, R., MCDOWELL, R.M., MORALES, R.A, An Overview of the Salmonella Enteritidis Risk Assessment for Shell Eggs and Egg Products, Risk Analysis, vol. 22, 2002, pp. 203 - 218.

21.GUARD-PETTER, J., The chicken, the egg and Salmonella entretidis, Environmental Microbiology, no. 3, 2001, pp. 421 - 430.

22. HERIKSTAD, H., MOTARJEMI, Y., TAUXE, R.V., Salmonella surveillance: a global survery of public health serotyping, Epidemiology and Infection, no. 129, 2002, pp. 1 - 8.

23.GAST, R.K., HOLT, P.S., Deposition of phage type 4 and 13a Salmonella entretidis strains in the yolk and albumen of eggs laid by experimentally infected hens, Avian Diseases, no. 44, 2000, pp. 706 - 710.

24.AHN, D., KIM, S., SHU, H., Effect of egg size and strain and age of hens on the solids content of chicken eggs, Poultry Science, vol. 76, no. 6, 1997, pp. 914-919.

25.ROSSI, M., CASIRAGHI, E., PRIMAVESI, L., POMPEI, C., HIDALGO, A., Functional properties of pasteurized liquid whole egg products as affected by the hygienic quality of the raw eggs, LWT - Food Science and Technology, vol. 43, no. 3, 2010, pp. 436-441.

26.FILIMON, V., BORDA, D., ALEXE, P., STOICA, M., Study of PATP Impact on Food Packaging Materials, Mat. Plast., 53, no. 1, 2016, p. 48 27.MILUTINOVIC, M., HADZISTEVIC, M., MOVRIN, D., VRBA, I., Non conventional Methods for Shaping Plastics Parts, Mat. Plast., 48, no. 3, 2011, p. 222

28.PIRINGER, 0.G., Prediction of Diffusion Coefficients in Plastic Materials, Rev. Chim. (Bucharest), 59, no. 11, 2008, p. 1186.

29.DAWSON, P.L., STEPHENS, C., Poultry Packaging-Poultry Meat Processing and Quality, Woodhead Publishing Limited, Cambridge, England, 2004, pp. 135-163.

30.HOTCHKISS, J.J., Food-packaging interactions influencing quality and safety, Food Addit Contam vol. 14, no. 6-7, 1997, pp. 601-607.

31.KIRWAN, M.J ., STRAW BRIDGE, J.W., Plastics in food packaging. In: Coles R, McDowell D, Kirwan MJ, editors. Food packaging technology. London, U.K.: Blackwell Publishing, CRC Press, 2003, pp. 174-240. 
32.LAU, O.W., WONG, S.K., Contamination in food from packaging materials, J. Chrom. A. vol. 882, no. 1-2, 2000, pp.255-270.

33.LOPEZ RUBIO, A., ALMENAR, E., HERNANDEZ-MUNOZ, P., LAGARON, J.M., CATALA, R, GAVARA, R., Overview of active polymerbased packaging technologies for food application, Food Rev. Int., vol. 20, no. 4, 2004, pp. 357-87.

34. *** REGULATION (RE) 89/437, STAS 2213/1-88.

35.DOLIS, M.G., SIMEANU, C., USTUROI, A., SIMEANU, D., Research Regarding Chemical Composition and the Digestibility of the Mulberry Leaves from Eforie Variety, Rev. Chim. (Bucharest), 68, no.1, 2017, p. 151

36.RADU-RUSU, R.M., USTUROI, M.G., LEAHU, A., AMARIEI, S., RADURUSU, C.G., VACARU OPRIS, I., Chemical features, cholesterol and energy content of table hen eggs from conventional and alternative farming systems, South African Journal of Animal Science, 44, no. 1, 2014, pp. $33-42$.

37.ASSOCIATION OF OFFICIAL ANALYTICAL CHEMISTS- AOAC, Official Methods of Analysis 15th Edition, Volume Two - Food Compositions; Additives; Natural Contaminants, edited by Kenneth Helrich, Published by the Association of Official Analytical Chemists, Inc., Arlington, Virginia. Solids (total) in eggs - 925.30, 1990, pp. 851

38.*** ASSOCIATION OF OFFICIAL ANALYTICAL CHEMISTS - AOAC, Official Methods of Analysis 15th Edition, Volume Two - Food Compositions; Additives; Natural Contaminants, edited by Kenneth Helrich, Published by the Association of Official Analytical Chemists, Inc., Arlington, Virginia. Nitrogen in eggs Kjeldahl Method - 925.31, 1990, pp. 854.

39.TORAN, A.A., BARBERA, R., FARRE, R., LAGARDA, M.J., LOPEZ, J.C., HPLC Method for Cyst(e)ine and Methionine in Infant Formulas. J. Food Sci., 1996, 61, no. 6, pp. 1132-1136.

40.OPIENSKA-BLAUTH, J., CHARINSKI, M., BERLIE, H., A new rapid method for the determination of tryptophan, Analytical Biochemistry, 1963, vol. 6, pp. $69-76$.
41.ASSOCIATION OF OFFICIAL ANALYTICAL CHEMISTS- AOAC, Official Methods of Analysis 15th Edition, Volume Two - Food Compositions; Additives; Natural Contaminants, edited by Kenneth Helrich, Published by the Association of Official Analytical Chemists, Inc., Arlington, Virginia. Fat in eggs Acid hydrolysis method 925.32, 1990, pp. 854 42.*** ASSOCIATION OF OFFICIAL ANALYTICAL CHEMISTS - AOAC, Official Methods of Analysis 15th Edition, Volume Two - Food Compositions; Additives; Natural Contaminants, edited by Kenneth Helrich, Published by the Association of Official Analytical Chemists, Inc., Arlington, Virginia. Fatty Acids in eggs, Gas Chromatographic method, pp. 860.

43.MACIUC, V., CREANGA, S. MACIUC, D., VIDU, L., A New Programe for Data Management in Dairy Farms. ST26733. International Conference Agriculture for Life, Life for Agriculture, Agriculture and Agricultural Science Procedia vol. 6, 2015, pp. 226-231.

44.HUANG, S., HERALD, T.J ., MUELLER, D.D., Effect of electron beam irradiation on physical, physicochemical, and functional properties of liquid egg yolk during frozen storage, Poultry Science, 76, 1997, pp. $1607-1615$.

45.HANK, C.R., KUNKEL, M.E., DAW SON, P.L., ACTON, J.C., WARDLAW, F.B. JR., The Effect of Shell Egg Pasteurization on the Protein Quality of Albumen, J ournal of Poultry Science, vol. 80, 2001, pp. 821-824. 46.HESHAM, M.B., Effect of gamma radiation and cold storage on chemical and organoleptic properties and microbiological status of liquid egg white and yolk, Food Chemistry 97, 2016, pp. 285 - 293. 47.SAUVEUR, B., Metode d'élevage des poules et qualité de l'oeuf de consummation. INRA Prod. Anim., no. 4, pp. 123 - 130.

48.NYS, Y., SAUVEUR, B., Valeur nutritionnelle des oeufs, INRA prod. Anim., vol. 17, no. 5, 2004, pp. 385 - 393.

$\overline{\text { Manuscript received: } 28.12 .2016}$ 